\title{
Acute Ischemic Stroke Secondary to Ulcerative Colitis Successfully Managed with Direct Oral Anticoagulant
}

Rahul Handa ( $\square$ rahulhanda0411@gmail.com )

BLK Super Speciality Hospital, New Delhi https://orcid.org/0000-0002-3400-5681

\section{Satyan Nanda}

BLK Super Speciality Hospital, New Delhi

\section{Atul Prasad}

BLK Super Speciality Hospital, New Delhi

Rajiv Anand

BLK Super Speciality Hospital, New Delhi

\section{Man Mohan Mehndiratta}

BLK Super Speciality Hospital, New Delhi

Dhruv Zutshi

BLK Super Speciality Hospital, New Delhi

Ajay Kumar

BLK Super Speciality Hospital, New Delhi

Amrish Sahney

BLK Super Speciality Hospital, New Delhi Jasbir Singh

BLK Super Speciality Hospital, New Delhi

\section{Sujata K Dass}

BLK Super Speciality Hospital, New Delhi

\section{Prabhjeet Kaur Bedi}

BLK Super Speciality Hospital, New Delhi

\section{Vishav Dev Singh Dadwal}

BLK Super Speciality Hospital, New Delhi

\section{Aarti Pahuja}

BLK Super Speciality Hospital, New Delhi

\section{Pankaj Kumar Shah}

BLK Super Speciality Hospital, New Delhi

\section{Bipan Sharma}

BLK Super Speciality Hospital, New Delhi

Nalin Malhotra 
BLK Super Speciality Hospital, New Delhi

\section{Research Article}

Keywords: Ischemic Stroke, Ulcerative Colitis, Direct oral anticoagulant, Dabigatran

Posted Date: September 13th, 2021

DOl: https://doi.org/10.21203/rs.3.rs-337510/v1

License: (c) (i) This work is licensed under a Creative Commons Attribution 4.0 International License. Read Full License 


\section{Abstract}

Ulcerative Colitis (UC) is an inflammatory disorder of gastrointestinal tract characterised by chronic inflammation with episodes of relapses and remission. Neurological complications are not as rare as once thought, with occurrence of ischemic stroke in patients with UC ranging from 1.2-6.4\%. Although, an association of ulcerative colitis and stroke has been reported in various studies, pathogenesis and factors underlying this association are still not clear. Further, no specific treatment guidelines are available for ischemic stroke occurring in subjects with ulcerative colitis. As majority of patients of ulcerative colitis are seen by gastroenterologist, it is very important for them to be aware of this extraintestinal complication as early diagnosis and correct treatment can reduce the high morbidity associated with stroke. We herein report a case of Acute ischemic stroke secondary to ulcerative colitis successfully managed with Direct oral anticoagulant (DOAC). This case signifies the importance to have high index of suspicion among neurologists, especially in patients of young ischemic stroke with history of bleeding in stools or evidence of iron deficiency anaemia with no apparent cause, as treatment of the primary disease can significantly reduce the chances of recurrent stroke. Successful management of ischemic stroke complicating ulcerative colitis with Dabigatran indicates the safety of DOACs in patients of UC although, a randomised controlled trial is required before the use of DOACs in patients of UC with acute ischemic stroke can be approved.

\section{Introduction:}

Ulcerative Colitis (UC) is an inflammatory disorder of gastrointestinal tract characterised by chronic inflammation with episodes of relapses and remission [1]. Neurological complications are not as rare as once thought, with occurrence of ischemic stroke in patients with UC ranging from 1.2-6.4\% [2]. Most of the studies have found a higher incidence of stroke in patients with ulcerative colitis than general population, with more certainty in age group less than 50 years [3, 4]. Although, an association of ulcerative colitis and stroke has been reported in various studies, pathogenesis and factors underlying this association are still not clear. Further, no specific treatment guidelines are available for ischemic stroke occurring in subjects with ulcerative colitis [1]. We herein report a case of ischemic stroke secondary to ulcerative colitis successfully managed with Direct oral anticoagulant (Dabigatran).

\section{Case Report:}

A 35-year-old male with no known comorbidities presented to the emergency of our hospital with complaints of sudden onset non progressive weakness of right upper limb for 5 days. Patient had history of chronic diarrhoea for 3-4 years, but was never investigated earlier. On examination, patient was hemodynamically stable with Glasgow Coma Scale (GCS) as E4V5M6. Neurological examination revealed weak right-hand grip with muscle power 4-/5 in the right upper limb as per Medical Research Council (MRC) grading. Rest of the neurological examination including sensory examination was normal. National Institute of Health Stroke Scale (NIHSS) score was 1. A clinical diagnosis of acute stroke was made and patient underwent Magnetic resonance imaging (MRI) of the brain to confirm the diagnosis. 
MRI revealed acute ischemic stroke in left fronto-parietal lobe (Fig. 1). Patient was started on aspirin and atorvastatin and was advised admission to evaluate for the aetiology of stroke. Routine blood tests showed microcytic hypochromic anemia with haemoglobin: $8.6 \mathrm{~g} / \mathrm{dL}$ (normal: 13.8-17.2); mean corpuscular volume (MCV): $68.3 \mathrm{fL}$ (normal: 80-95 fL); mean corpuscular hemoglobin (MCH): $20.7 \mathrm{pg}$ (normal: $27.5-33.2 \mathrm{pg}$ ) with elevated platelet count: $646000 / \mu \mathrm{L}$ (normal: $150000-400000 / \mu \mathrm{L}$ ). Prothrombin time (PT) and activated partial thromboplastin time (aPTT) values were within normal range. Iron studies revealed iron deficiency anemia with serum Ferritin: $4.26 \mathrm{ng} / \mathrm{mL}$ (normal: $21-274$ $\mathrm{ng} / \mathrm{mL}$ ); serum Iron: 28.34 microgm/dL (normal: 59-158 microgm/dL); Transferrin Saturation: 9.60\% (normal: $30-50 \%)$; TIBC: $295 \mathrm{mcg} / \mathrm{dL}$ (normal: $240-450 \mathrm{mcg} / \mathrm{dl}$ ) and normal reticulocyte count: $1.9 \%$ (normal: 1-3\%). Inflammatory markers were elevated; ESR: $25 \mathrm{~mm} / \mathrm{hr}$ (normal: 1-10 mm/hour) and Creactive protein: $7.14 \mathrm{mg} / \mathrm{L}$ (normal $<5 \mathrm{mg} / \mathrm{L}$ ). Rest of the routine blood examination including liver function tests, kidney function tests, lipid profile and glycosylated haemoglobin were normal, except low serum albumin: $3 \mathrm{~g} / \mathrm{dl}$ (normal- 4.0 and $5.4 \mathrm{~g} / \mathrm{dl}$ ). Viral markers including Human Immunodeficiency Virus (HIV), Hepatitis B Surface antigen (HBsAg) and Hepatitis C Virus (HCV) antibody were negative. Workup for young ischemic stroke revealed elevated serum homocysteine levels: $21.43 \mathrm{micromol} / \mathrm{L}$ (normal: $<15$ micromoles/L) with negative antinuclear antibody (ANA) and Lupus Anticoagulant. Serum protein C, protein $\mathrm{S}$ and antithrombin III levels were normal, and there was no factor V Leiden mutation found. Perinuclear anti-neutrophil cytoplasmic antibodies ( $\mathrm{p}$-ANCA) was positive with negative cytoplasmic antineutrophil cytoplasmic antibodies (C-ANCA). Computed tomography angiogram (CTA) of brain and neck was normal. Cardiac evaluation including electrocardiogram (ECG), echocardiography and 24-hour Holter were normal. Digital subtraction angiography preformed to rule out small vessel vasculitis and dissection was essentially normal. Cerebrospinal fluid (CSF) Cells, Proteins and Sugar (including blood-CSF ratio) were normal. In view of chronic diarrhoea, microcytic hypochromic anaemia and hypoalbuminemia, a gastroenterologist opinion was sought to look for any site of occult bleeding or malabsorption and was advised upper and lower gastrointestinal tract (GI) endoscopy. Upper GI endoscopy was normal, but Colonoscopy revealed evidence of Inflammatory Bowel disease with ulcers and erosions involving the entire length of the colon and biopsy taken from these lesions was suggestive of active ulcerative colitis (Figs. 2 and 3). As UC is known to be associated with vascular complications due to hypercoagulable state, patient was advised D-dimer and Factor VIII assay. D-dimer was elevated: $2.16 \mathrm{mcg} / \mathrm{ml}$ (normal < $0.5 \mathrm{mcg} / \mathrm{ml}$ ) and so was Factor VIII assay: $245 \%$ (normal: $50-150 \%$ ). As there was evidence of hypercoagulable state (elevated D Dimer; elevated Factor VIII levels with thrombocytosis) and evidence of increased disease activity (raised ESR and CRP), patient was started on mesalamine, oral steroids, anticoagulation, folic acid, vitamin B6 and B12 and injectable iron. Thus, we considered the likely diagnosis of ischemic stroke secondary to hypercoagulable state due to active ulcerative colitis. Patient at the time of discharge i.e., five days after admission, had significant improvement in right hand grip and power in proximal right upper limb had improved to 4+/5 (MRC grading). Patient at discharge was advised Dabigatran, mesalamine, tapering dose of prednisolone, vitamin B6, vitamin B 12, folic acid and iron with other supportive treatment including physiotherapy. Patient at 2 weeks follow up had no neurological deficits and his haemoglobin had improved to $9.5 \mathrm{~g} / \mathrm{dl}$, and haemoglobin at 6 weeks follow up was $11 \mathrm{~g} / \mathrm{dl}$ with no fresh episode of blood in stools. 


\section{Discussion:}

Ulcerative colitis is more frequently associated with venous thrombosis including deep vein thrombosis and pulmonary embolism as compared to arterial infarcts including ischemic stroke. Although, exact cause of increase incidence of stroke in ulcerative colitis is not known, but hypercoagulable state as a possible mechanism is widely accepted [5]. Various studies have shown thrombocytosis, raised factor $\mathrm{V}$, factor VIII, factor IX, fibrinogen and D-Dimer in patients of ulcerative colitis presenting as ischemic stroke $[1,5]$. Presence of hypercoagulable state seems to be uniquely associated with inflammatory bowel disease (IBD) as it is not seen in other inflammatory conditions like rheumatoid arthritis or coeliac disease [6]. Our patient had elevated factor VIII and D-Dimer levels which favoured a hypercoagulable state and thus, was managed with anticoagulant rather than antiplatelet.

Although, risk factors leading to stroke in patients of ulcerative colitis are not fully known but an association with severity of disease, extent of colonic involvement, age of the patient and acquired hyperhomocysteinaemia secondary to vitamin B 6 and B 12 deficiency have all been reported in the past [6]. Approximately $30-40 \%$ of the patients of IBD presenting with stroke were found to have thrombocytosis and anaemia at presentation [2]. Although, most of the cases of ischemic stroke have been reported in patients with active ulcerative colitis, few cases have been reported even while in remission [6]. Our patient was a young male with no other comorbidity, had pan-colonic involvement, hyper homocysteinemia and evidence suggestive of active disease.

No specific guidelines for secondary prophylaxis of ischemic stroke in patients with ulcerative colitis is available. Aspirin and anticoagulants have been used in the past with varying success [1]. Treatment of ischemic stroke with antiplatelets or anticoagulants in patients of ulcerative colitis is a double-edged sword as it can increase the chances and severity of bleeding. Due to hypercoagulable state seen in ulcerative colitis with increase disease activity, prophylactic use of low molecular weight heparin to prevent vascular complications has been advised by British Society of Gastroenterology, as it was found to be safe in various studies, but the exact risk of bleeding with therapeutic dose of anticoagulants is not known [7]. We gave low molecular weight heparin followed by dabigatran in our patient as he had elevated D-dimer and factor VIII levels which were suggestive of hypercoagulable state. A few case series in the past have reported anticoagulant use in therapeutic dose in patients of ischemic stroke complicating UC, but only a handful of cases have been reported in literature in which Direct oral anticoagulants (DOACs) have been used $[1,2,6]$. We also prescribed him mesalamine for treatment of ulcerative colitis, vitamin B 6, vitamin B 12 and folic acid for hyper homocysteinemia and oral iron to treat anaemia. At 6 weeks follow up his haemoglobin had improved to $11 \mathrm{~g} / \mathrm{dl}$ and he had no history of blood in stools. Our plan is to repeat platelet count, ESR, CRP and D-dimer after 12 weeks of therapy and to convert dabigatran to aspirin if inflammatory markers and disease activity is found to be reduced.

\section{Conclusion:}


Vascular complications in ulcerative colitis are well known, but ischemic stroke is relatively rare. Ischemic stroke in UC is usually associated with high disease activity and hypercoagulable state seen in UC and thus, treatment involves management of primary disease along with antiplatelet or anticoagulant. As majority of patients of ulcerative colitis are seen by gastroenterologist, it is very important for them to be aware of this extra-intestinal complication as early diagnosis and correct treatment can reduce the high morbidity associated with stroke. This case signifies the importance of having high index of suspicion among neurologists, especially in patients of young ischemic stroke with history of bleeding in stools or evidence of iron deficiency anaemia and features of malabsorption with no apparent cause, as treatment of the primary disease can significantly reduce the chances of recurrent stroke. Successful management of ischemic stroke complicating ulcerative colitis with dabigatran indicates the safety of DOACs in patients of UC although, a randomised controlled trial is required before the use of DOACs in patients of UC with acute ischemic stroke can be approved.

\section{Declarations:}

\section{Funding source:}

We received no grant for this case.

\section{Declaration of competing interest:}

There is no financial and intellectual conflict of interest.

\section{Availability of data and material:}

The datasets generated during and/or analyzed during the current study are available from the corresponding author on reasonable request.

Code availability: Not applicable

Ethics approval: Not applicable

Consent to participate: Yes. The authors received written consent from the patient for participation.

Consent for publication: Yes. The authors affirm that the patient has consented to the submission of the case report for submission to a scientific journal. Moreover, the authors affirm that the patient provided written informed consent for publication of the images

\section{Authors' contributions:}

First author:: Writing of the first draft.

All authors: Review and critique of the manuscript 


\section{References:}

1. Shaban A, Hymel B, Chavez-Keatts M, Karlitz JJ, Martin-Schild S. Recurrent posterior strokes in inflammatory bowel disease patients. Gastroenterol Res Pract. 2015;2015:672460.

2. Katsanos AH, Kosmidou M, Giannopoulos S, et al. Cerebral arterial infarction in inflammatory bowel diseases. Eur J Intern Med. 2014;25(1):37-44.

3. Singh S, Singh H, Loftus EV Jr, Pardi DS. Risk of cerebrovascular accidents and ischemic heart disease in patients with inflammatory bowel disease: a systematic review and meta-analysis. Clin Gastroenterol Hepatol. 2014;12(3): 382-93.e1: quiz e22.

4. Andersohn, M. Waring, and E. Garbe, "Risk of ischemic stroke in patients with Crohn's disease: a population-based nested case-control study," Inflammatory Bowel Diseases, 2010; vol. 16, no. 8, pp. 1387-1392.

5. Jorens P G, Delvigne C R, Hermans C R, Haber I, Holvoet J, De Deyn P P. Cerebral arterial thrombosis preceding ulcerative colitis. 1991; 22 (9) 1212

6. Joshi D, Dickel T, Aga R, Smith-Laing G. Stroke in inflammatory bowel disease: a report of two cases and review of the literature. Thromb J. 2008;6:2.

7. Irving PM, Pasi KJ, Rampton DS. Thrombosis and inflammatory bowel disease. Clinical Gastroenterology and Hepatology : the Official Clinical Practice Journal of the American Gastroenterological Association. 2005 Jul;3(7):617-628.

\section{Figures}

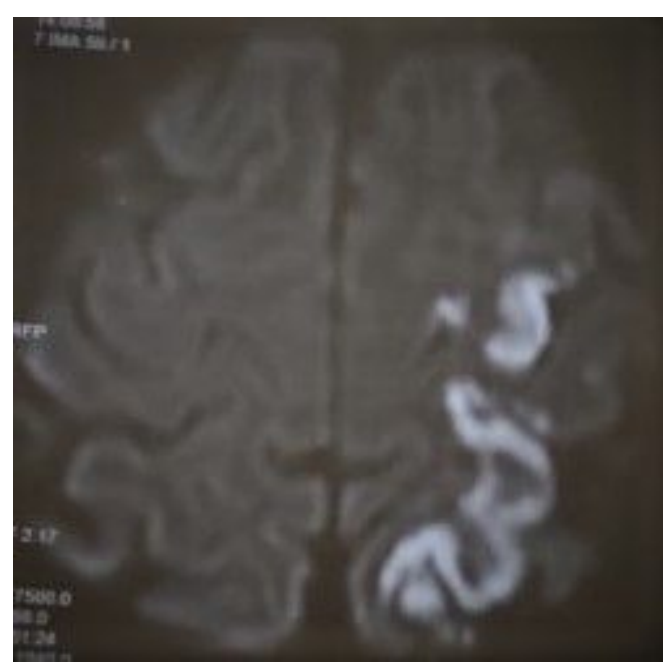

Figure 1

MR DW image showing diffusion restriction suggestive of left fronto-parietal acute infarct 


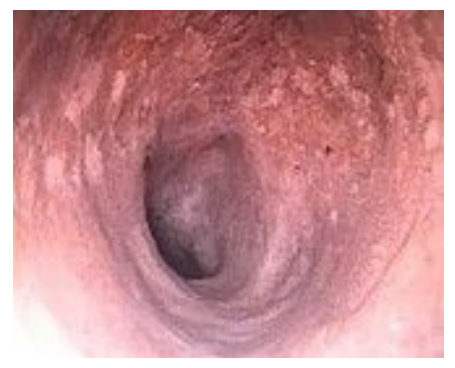

Figure 2

Colonoscopy showing superficial ulceration and complete obliteration of vascular pattern and fine granular appearance of mucosa of rectum 


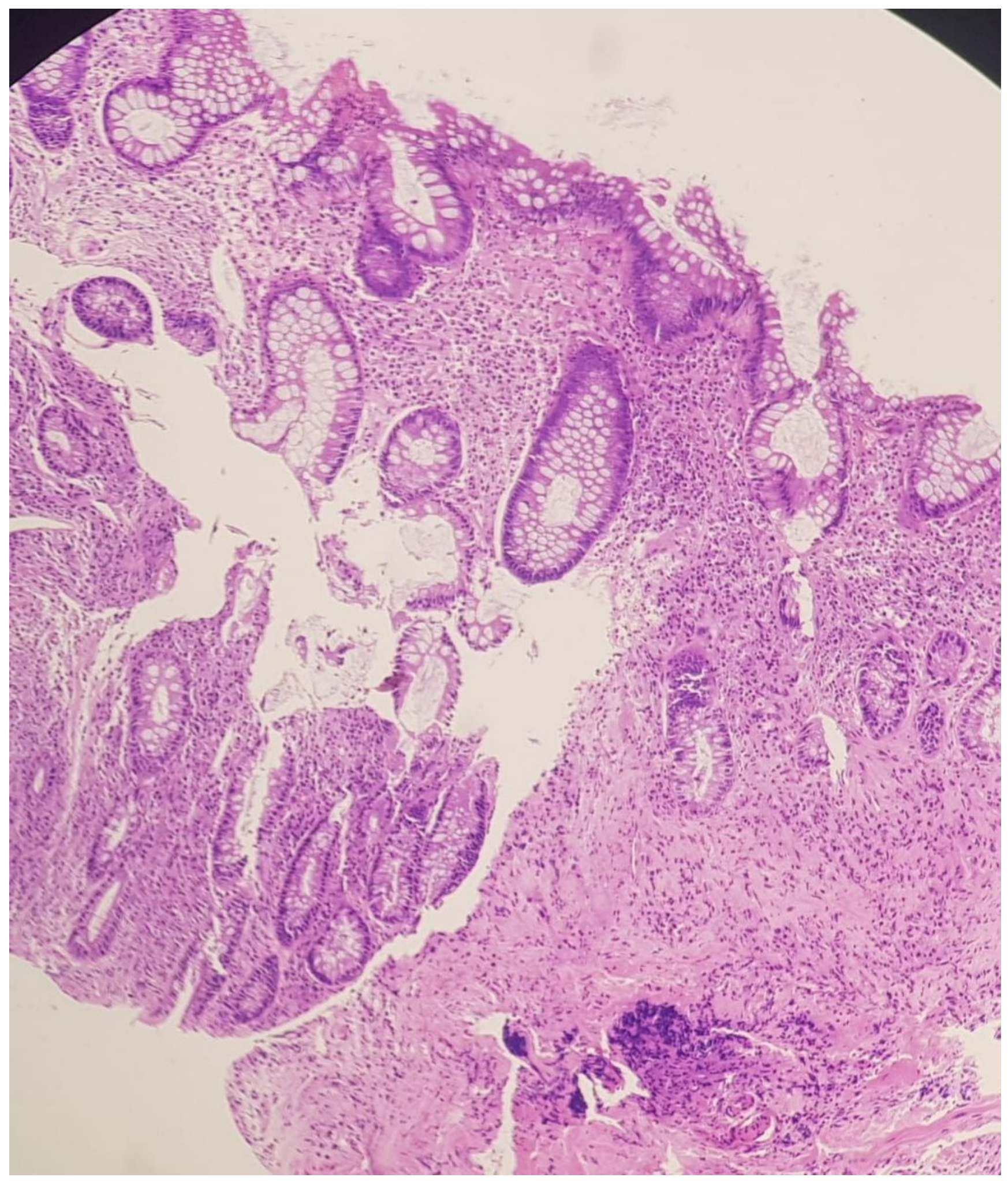

\section{Figure 3}

H\&E stained section of large intestine mucosa showing crypt architecture distortion, crypt branching, crypt drop outs and basal plasmacytosis with cryptitis and crypt abscess suggestive of chronic active inflammation. 